\title{
Research of wood waste concentration influence on composite fuel energy characteristics based on low-grade 2 b coal from "Borodinskoe" deposit
}

\author{
$S$. Yankovskiy ${ }^{1, *}, A$. Matveev ${ }^{1}, A$. Tolokolnikov ${ }^{l}$, and $A$. Zenkov \\ ${ }^{1}$ National Research Tomsk polytechnic university, 634050, Tomsk, Russia
}

\begin{abstract}
A pyrolysis process of different coal samples into three different types of oxidizing medium (argon, air and steam) were studied by means of thermogravimetry. Two coal types with different metamorphism degree (lignite and bituminous coal) were used. The experimental procedure was carried out in non-isothermal conditions in temperature range from $373 \mathrm{~K}$ to $1273 \mathrm{~K}$ with $20 \mathrm{~K} / \mathrm{min}$ heating rate. Purge gas consisted of argon and oxidizer with volumetric ratio 1:24 and had 250 $\mathrm{ml} / \mathrm{min}$ flow rate. Kinetic parameters were defined by fitting procedure using Arrhenius-type equation with the first order reaction rate on conversion distribution function. The ignition and burnout indexes were calculated to evaluate sample reactivity at different oxidizing mediums. The highest reactivity values in same atmosphere were obtained for lignite because of higher particle special surface area. For same samples the higher reactivity was obtained in steam medium. The conclusion about some kind of synergy between carbon oxidation and pyrolysis could be made.
\end{abstract}

\section{Introduction}

Production capacity of timber industry increases every year in Russian Federation [1]. Results of quality products production are accompanied by a parallel increase in the amount of wood waste in the form of sawdust, bark, wood chips and veneer shorts [1], conservation of which poses a threat to the environment [1].

In turn, the use of wood waste in small-scale power generation is not highly relevant because of the resulting materials low calorific value and applied rarely.

The process of concentration influence of one fuel or another in a mixed composition on calorific value, ash content, volatile substances release and combustion kinetics is poorly researched. Application of this composition in the form of briquettes leads to a decrease of the combustion temperature, ash entrainment, consumption of traditional fuels in decentralized areas and an increase of energy equipment efficiency. As a result, transportation component is reduced, and the use of local fuel will lead to synergistic effect.

* Corresponding author: jankovsky@tpu.ru 


\section{Experimental research}

The researched samples preparation was carried out by milling followed by sieving. Particle size of investigated coal powder was less than 80 microns, timber - less than 200 microns $[2]$.

At the preliminary stage of primary fuels gasification research, their element analysis was defined with the help of scanning electron microscope (CЭM) JEOL JCM-6000 [3]

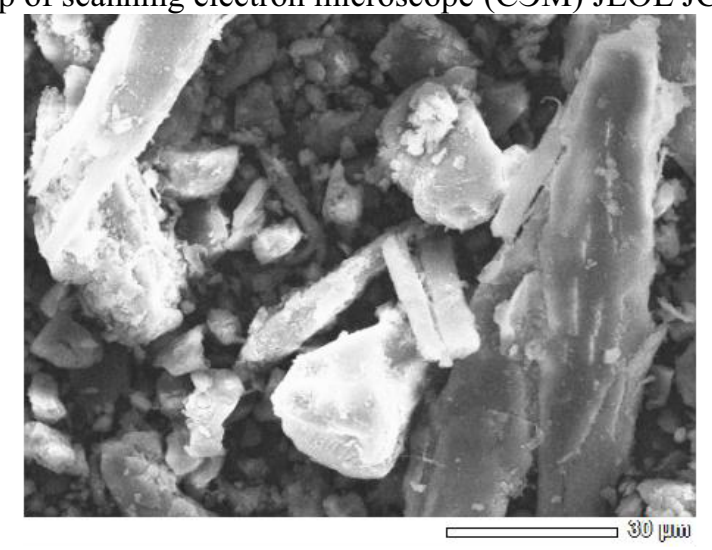

Fig. 1. The figure shows an image of the composite fuel with an increase in $30 \mu \mathrm{m}$.

The elemental composition of the composite fuel is shown in Figure 2.

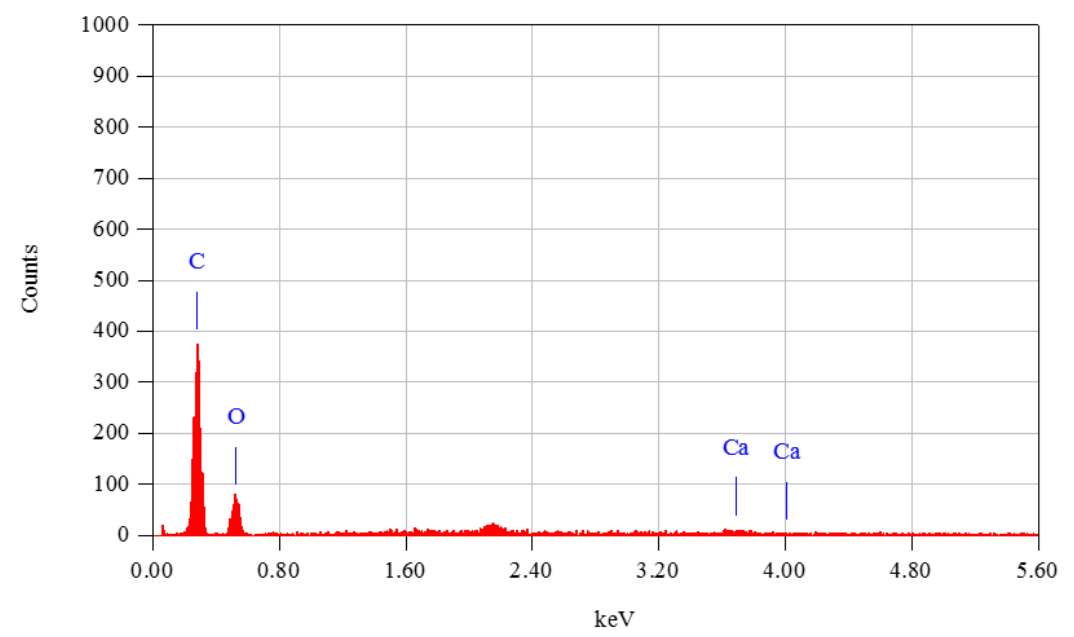

Fig. 2. The elemental composition of the composite fuel.

These milled 2B coal samples (Borodinskoe deposit) and timber production waste were mixed in Pulverisette 6 planetary mill [4-5] in the proportions of $10 \%$ of sawdust, 90 $\%$ of coal; $25 \%$ of sawdust, $75 \%$ of coal; $50 \%$ of sawdust, $50 \%$ of coal; $75 \%$ of sawdust, $25 \%$ of coal; $90 \%$ of sawdust, $10 \%$ of coal. Technical analysis of obtained mixed fuel samples was conducted.

As a result of a series of experiments, dependence of mixed fuel calorific value on its composition was obtained. 


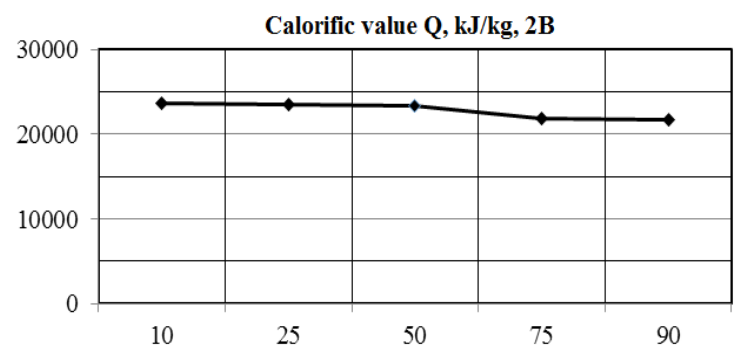

Fig. 3. Dependence of composite fuel calorific value from the percentage ratio of the primary fuel and timber production waste.

Figure 3 shows that mixing of $2 \mathrm{~B}$ grade coal with fine timber in $50 / 50$ percentage ratio reduces total calorific value by $2 \%$, and the concentration of $90 / 10 \%$ leads to a reduction of calorific value by $8 \%$.

The dependences of ash content and volatile substances release changes of mixed fuel from its composition are obtained.

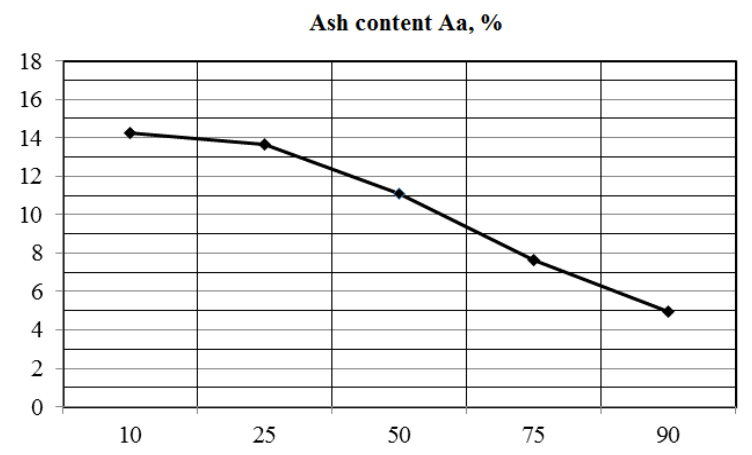

Fig. 4. Changes in ash content release of composite fuel from the percentage ratio of the main fuel and timber waste.

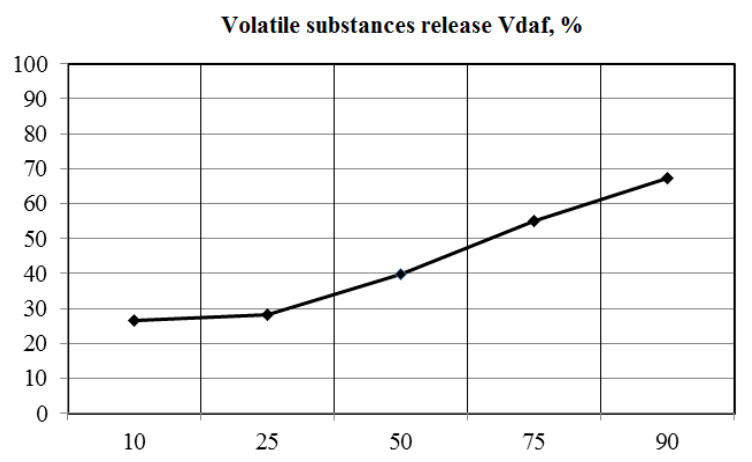

Fig. 5. Changes in volatile content substances release of composite fuel from the percentage ratio of the main fuel and timber waste.

Figure 4, 5 shows that ash content is considerably reduced with an increase of wood concentration: ash content drops to $75 \%$ at $90 \%$ concentration of timber within the initial fuel. An increase of wood content in composite fuel based on 2B grade coal increases the volatile substances release up to $41 \%$. 


\section{Conclusion}

The obtained results allow us to determine fine timber (wood industry waste) concentration effect on the kinetic parameters of 2B grade coal. It is revealed that an increase in wood concentration in the fuel reduces the ignition temperature, activation energy and increases combustible gases intensification. Calorific value varies within $2 \%$ when sawdust concentration in mixed fuel is changed from $10 \%$ to $50 \%$.

The work has been supported by the Russian Federal focused program "Research and development on priority orientation of science and technology in Russia 2014-2020", unique identifier 'Applied Research and Experimental Developments' project RFMEFI58114X0001.

\section{References}

1. Doklad o povishenii effektivnosti lesnogo kompleksa [Report on improving the effectiveness of timber complex], 138 (2013)

2. S. Jankovskiy, D. Luzhkovoj, K. Larionov, A. Matveeva, MATEC Web Conf. 37, 4 (2015)

3. Dzh. Goulstejn, Rastrovaja jelektronnaja mikroskopija i rentgenovskij mikroanaliz (Mir, Moskva, 1984)

4. A. Zenkov, S. Yankovsky, A. Matveeva, S. Lavrinenko, G. Alexander, MATEC Web Conf. 72, 1131 (2016)

5. A.G. Korotkikh, K.V. Slyusarskiy, MATEC Web Conf. 23, 01020 (2015) 